\title{
Evolutionary understanding of the conditions leading to estimation of behavioral properties through system dynamics
}

\author{
Chulwook Park ${ }^{1,2^{*}}$ (1)
}

\author{
${ }^{*}$ Correspondence: \\ pcw8531@gmail.com; \\ park@iiasa.ac.at \\ ${ }^{1}$ International Institute \\ for Applied Systems Analysis \\ (IIASA), 2361 Laxenburg, \\ Austria \\ Full list of author information \\ is available at the end of the \\ article
}

\begin{abstract}
One of the basic approaches in science views behavioral products as a process within a dynamic system. The mechanism might be seen as a representation of many instances of centralized control in real time. Many real systems, however, exhibit autonomy by denying statically treated mechanisms. This study addresses the issues related to the identification of dynamic systems and suggests how determining the basic principles of a collective structure may be the key to understanding complex behavioral processes. A fundamental model is derived to assess the advantages of this perspective using a basic methodology. The connection between perspective and technique demonstrates certain aspects within their actual context while also clearly including the framework of actual dynamic system identification.
\end{abstract}

Keywords: System approach, Agent-based model, Elementary coordination, Circadian cycles, Collective structure

\section{Introduction}

System dynamics can be used as a tool to address many applications in a very broad sense. It applies to systems in many different disciplines, such as control, communication, tropisms, and even systems of systems (Mandl 2019). Given the very widespread use of the term "system," the important questions are as follows: What does the term "system" actually mean? What makes a system "dynamic"? These terms cannot be defined through strict codification because a system is an abstract or vague entity (Rosen 1985). A well-formulated notion of a system could, however, be developed using a nonsystem definition. A nonsystem can be represented by a set of isolated entities that do not interact with each other or a collection of entities whose relationships have no implications for the properties or behaviors of the entities (Pattee 1973). Given how vague word definitions can be, the notions that we use describe a wide variety of things (Marchal 1975). Let us thus specify how these notions may be understood in the light of an account of a dynamic system.

(c) The Author(s) 2020. This article is licensed under a Creative Commons Attribution 4.0 International License, which permits use, sharing, adaptation, distribution and reproduction in any medium or format, as long as you give appropriate credit to the original author(s) and the source, provide a link to the Creative Commons licence, and indicate if changes were made. The images or other third party material in this article are included in the article's Creative Commons licence, unless indicated otherwise in a credit line to the material. If material is not included in the article's Creative Commons licence and your intended use is not permitted by statutory regulation or exceeds the permitted use, you will need to obtain permission directly from the copyright holder. To view a copy of this licence, visit http://creativeco mmons.org/licenses/by/4.0/. 
First, a system is a collection of individual elements: it is not usual to talk about a system having only one component (Chen and Billings 1992). It is then likely that the elements comprising the system exhibit nontrivial interactions, for instance, coherence in terms of moving or working together $\left[\chi \rightarrow \vec{f} \rightarrow f\left(x_{i}\right)\right]$. Here, $x$ denotes an element composed of many individuals; $f$ is a function toward a certain direction $(\rightarrow)$, such as a goal, and $f\left(x_{i}\right)$ denotes coherence, which involves certain individuals $\left(x_{n}\right)$, but not all individuals, underlying a very well-defined goal boundary $(f)$. The second importantindeed, key-assumption for a system is that the well-defined individuals must interact not just with one another but also with everything outside the system (Schöner 2002), which is called the environment $\left[x_{t} \rightarrow f\left(x_{i}\right) \rightarrow y_{t}\right]$. In this equation, there are two kinds of interactions that $x_{i}$ can have with the outside environment. The outside exerts some impact $(x)$ on them and is influenced $(y)$ by them at a certain time $(t)$. Thus, intuitively, a system can be defined as many interactive individual and embedded elements exhibiting a certain coherent behavior (Barabasi 2005).

Likewise, if we think about the system at any given time as defined as a set of numbers that are the values $\left(x_{i}\right)$ of variables, the collection of a system will be defined as identical to, and specified by, the functional forms $f_{i}$ over time ( $\mathrm{t}$ ) from time $=0$ $\left[\frac{d x_{i}}{d t}=f_{i}\left(x_{1}, x_{2}, \ldots, x_{n}\right), \quad(i=1,2, \ldots, n)\right]$. However, no behavior of the system can correspond to this logic, as no other systems' behaviors are constant in time. In other words, systems are such that behaviors occur over different timescales, in the same way that the behaviors of a group of people while ice skating occur over different timescales (Borgonovi et al. 2019): every event should be the initial condition for the next slice of time, and every division should set the stage for the next. The coherence of performance of the people (on the ice) must be nested dynamically within a continuous time interval (Chen and Billings 1992). Thus, if we consider that there are certain continuous approximations using finite intervals of time between changes of state within an interval, the system can be absorbed into a new functional type of organization $\left[\frac{d x_{i}}{d t}=g_{i}\left(x_{1}, x_{2}, \ldots, x_{n}-1\right), \quad(i=1,2, \ldots, n-1)\right]$.

In this logic, the system contains two variables, each of which is absolute but each of which changes suddenly to the other in the field (Schöner 2002). This may be a product of multiple causes by which any individual changes its behavior dynamically (Ashby 1947). At this point, the questions of "What is a system?" and "What makes a system dynamic?" seem to be recognized with regard to the picture, and the situation seems far less static.

\section{Part 1: Nonrepresentational perspectives of phenomena (theoretical basis)}

There is a well-defined assumption called the problem of impoverished entailment (Shaw 2001), which is the minimal starting point for understanding any system of interest at any level of interest. " $\mathrm{X}$ is about $\mathrm{Y}$ " is true only if " $\mathrm{X}$ entails $\mathrm{Y}$ " and " $\mathrm{Y}$ entails $\mathrm{X}$ " are true. This is a loop of entailment; the specificity of $X$ to its source of $Y$ means that $\mathrm{X}$ entails the source by which $\mathrm{X}$ is entailed. Diagrammatically, an entailment can be expressed as $\mathrm{X} \rightarrow \mathrm{Y}$. The primary property of an entailment is that it propagates "truth" hereditarily $-Y$ inherits the truth of X (Rosen 1991). Thus, the loop of entailment can be shown diagrammatically as $\mathrm{X} \rightarrow \mathrm{Y} \rightarrow \mathrm{X}$, and the diagram can be read as meaning that truth propagates hereditarily in both directions. Such an explanation focuses on assembling all parts of elements $[C(s)]$ along with the other "things" that can influence them 
or be influenced by them $[E(s)]$, even the internal structure of the system $[S(s)]$ (Mahner and Bunge 1997).

$$
m(s)=\langle C(s), E(s), S(s)\rangle .
$$

In this framework, when $m$ is predicated as a mass of a particular object, it identifies a substantial property intrinsic to the object that is identical whenever and wherever the object is observed. However, $m$ has other relational properties that engage actual things. For example, when the object is grasped and brought down hard and repetitively on another object, then $m$ can be a "hammer" with respect to the object. Conceivably, there are many relational properties that the object may have by virtue of its relationship either with other objects or with perceivers-actors, but these are, at best, indefinite properties until a particular spatiotemporal relationship is effected (Gibson 1979). When this occurs, one of the many potential relational properties of the object is actualized.

However, investigating the dynamics in these cases typically requires a significant number of elements and obviously includes multiple components that must be managed. According to researchers (Bernstein 1966), organisms have too many degrees of freedom. Moreover, if the parts are considered to be very strongly defined by their connections and to function within the context, great complexity can be observed (Rosen 1987). When even a single cell's behavior is being considered, its tendencies do not allow certain prediction of all of these dynamics (Ford 2008; Strong and Ray 1975), and this prompts deep concerns over how to treat the cell relevantly as well as raising questions about how these behaviors form from numerous factors.

Instead of positively interpreting the system's dynamics in terms of every encoded equation, the key idea is to describe why numerous modes of emergent phenomena underlying local-level interaction have to be governed by simple rules (Iberall and Soodak 1987). Where elements are given a simple set of rules that govern their behavior and allow them to interact to determine what patterns emerge over time, it has been shown that an agent's behavior with respect to unpredictable phenomena can arise even with elementary governing rules (Roundy et al. 2018). This fresh perspective on refocusing a system's dynamics is helping to bridge traditional biases and has been stimulating scientists to distil out simple principles, such that a better understanding of a dynamic system (which is not always very complicated) may be gained.

\section{Part 2: Individual behavior in social dynamics (model-based)}

To explore the rule of thumb from a broader perspective, computer simulation has been performed first on the basics of a spatially explicit model of mobile agents in continuous space (Rangel et al. 2018) to determine the basic regulatory principles involved in the way they conceptualize their environment (Bonabeau 2002). In other words, the implemented model shows the potential to infer how simple individual rules can lead to consistent group behavior and how slight changes in those mechanisms can have a dramatic impact on an individual's behavioral patterns (Reynolds 1987). Although an analysis of simple implication is an apparent first step in providing proof of concept, this individual-based simulation has become significant enough to be tested in a broader range of applications for evolutionary dynamics for the following reasons. First, as the agents 
represent individuals who have participated from the bottom up, the actual state of their behavior tends to be more informative (agent-based modeling) than in other cases. Next, as the main point of this implementation is a description that deals with the state per time as the critical factor in its allocation of neighbors, the number of neighbors placed on the position is based on the moves scheduled for a given moment (context-varying cultural evolution) (Oliva 2016) [see "Methods I (agent-based model)" for more detail].

Result

The model provides a natural description of a pattern of behavior and allows us to understand a realistic adaptation incorporating behavioral algorithms with social dynamics. The mechanisms characterized in the agenda show that the model has the capacity to produce three types of factors. First, the behavioral pattern is the result of the applied individual components; this comes not only from the initial conditions of the autonomous agents but also from the fact that they are interconnected. Second, the range of

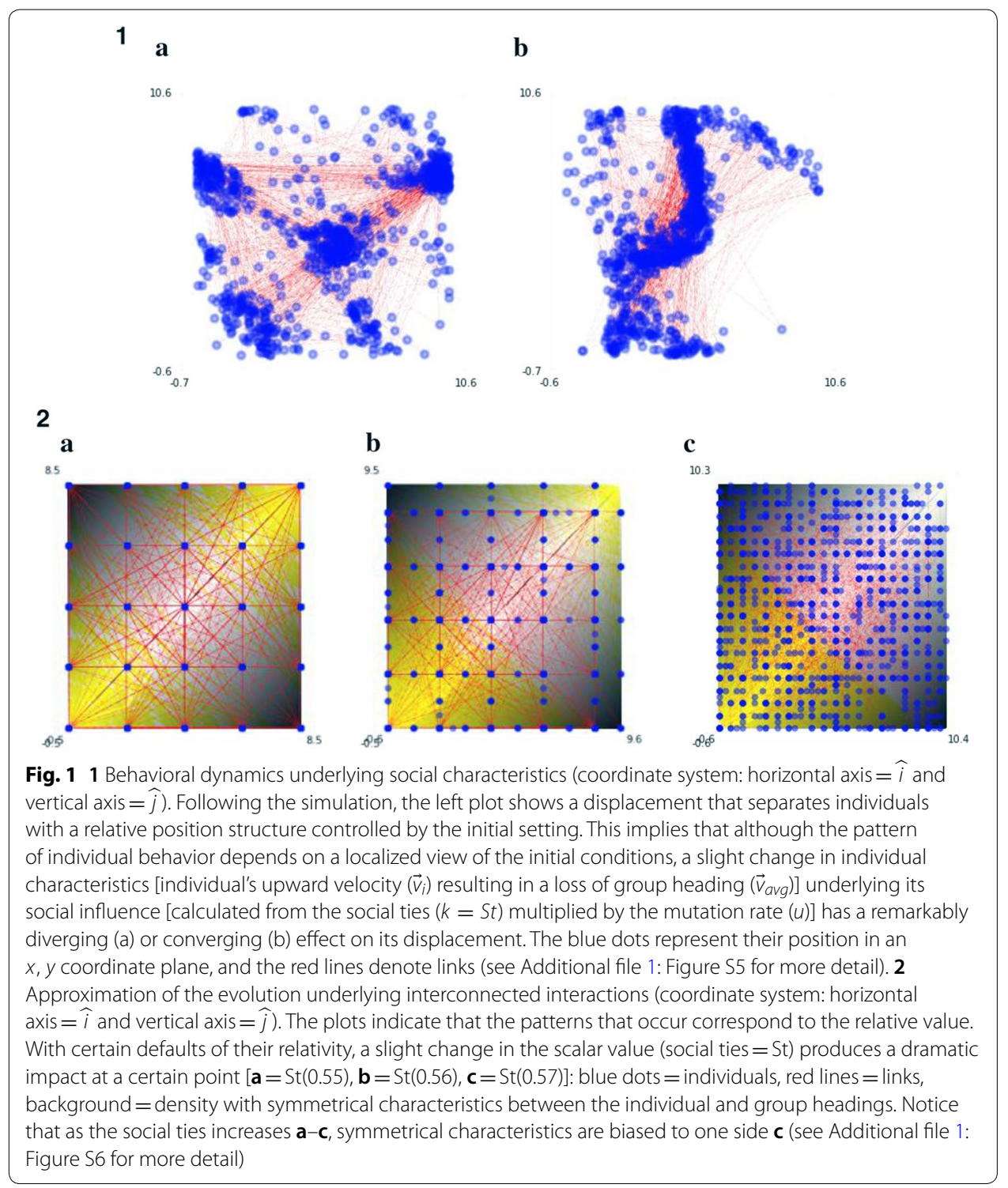


different combinations of the internal and the external states plays a part in the rapid propagation in the system (see Fig. 1-1). Third, however, when the interconnected relation between the internal trait underlying its external trait is applied, the widespread heterogeneity of the mechanisms can abstract the repertoire of displayed behaviors (see Fig. 1-2) (see Additional file 1: 1.4 for more detail).

The primary feature of the agents' interactions is heterogeneous in this abstract setting. As the topology of the interaction traits can lead to significant deviations from the predicted pattern of behavior, it may generate various effects that mimic the behavior of real individuals in social dynamics. At the points where these individuals interact, sensible decisions occur, in line with an empirical study showing that individuals learn how to keep relative velocity as a key factor for homogeneity (McLeod and Dienes 1996).

\section{Discussion}

The simulations show that different interconnection structures have an effect on which strategies perform better, a relationship referred to as ecological rationality. The results may suggest that the relative velocity can be the pure candidate and works effectively. According to researchers (McLeod and Dienes 1996), if an object (i.e., a ball) is already high in the air and travelling directly in line with the individual (i.e., the player), the individual might utilize some simple heuristics. Namely, the individual fixes his gaze on the object, starts running, and adjusts his velocity to ensure that the angle of the ball above the horizon appears constant (Gigerenzer 2004). The prediction is not that the individual runs to a precomputed landing spot and waits for the object but that he modifies his actions to keep the image of the object moving at a constant velocity. It is also possible that individuals do not compute $(\vec{v})$ at all in this model but would reduce a maintained value of $d^{2}(\vec{v}) / d t^{2}$ in a systematic way. As $\vec{v}$ increased, they would keep $d^{2}(\vec{v}) / d t^{2}$ at zero $\left[d^{2}(\vec{v}) / d t^{2}=\right.$ constant] (Reed et al. 2010). This is related to what information can be derived as a strategy in the system and how that information can be best obtained through the dynamics.

This result also holds for a step progression toward such a relationship, as explicitly shown by the dramatic change in regard to the point where $\left[d^{2}(\vec{v}) / d t^{2}=\right.$ constant $]$ reaches $\left[d^{2}(\vec{v}) / d t^{2} \neq\right.$ constant $]$. Let us suggest that actualized observation of the pattern corresponds not simply to the object's velocity but, instead, to changes in the velocity between individuals at a certain point. In other words, the actual displacement $(S)$ estimated is given by $\left(S_{l^{1}}=S_{l}+\alpha d^{2}\right)$. Here, the observed new displacement $\left(S_{l^{1}}\right)$ is equal to the displacement across the individuals $\left(l^{1}\right)$ plus its relative velocity $(\alpha)$ multiplied by the distance squared $\left(d^{2}\right)$ from the neighbor. This indicates that the farther an individual is from the neighbor (role model or group heading), when measured at a certain point, the more difficult it is for the individual to follow the neighbor because of the greater acceleration involved. On this assumption, the results might suggest a strategy such as evolvable traits or payoff functions to guide the evolution of these heuristics through social learning (see Fig. 2). 


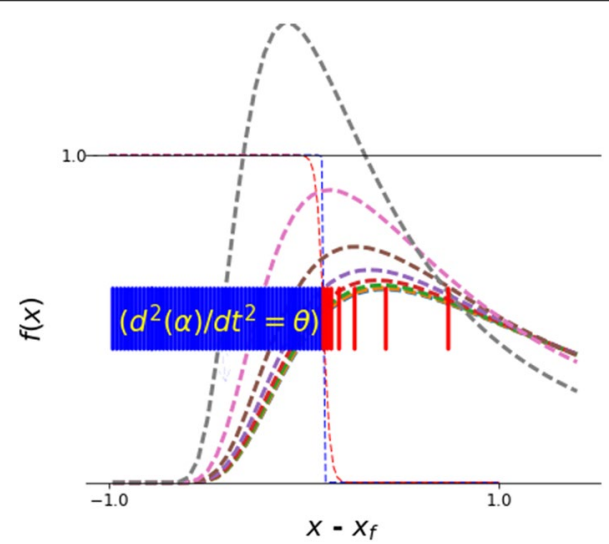

Fig. 2 Heuristics through the individual-based model. Based on the relativity defaults set by the model as an interconnected condition, the system becomes highly sensitive to small changes in the scalar values (i.e., social ties) of individuals at a certain point. The horizontal axis of the normal distribution denotes a scalar [ $(x$ ) = social ties in this simulation) from 0 to 1 , and the vertical axis represents the probability density at the scalar value $(x)$. This suggests that if the individual fails to keep the trait (blue area $=$ range from St $0.55 \rightarrow$ ) about the nearby individual, the displacement (red bars) will exponentially decay (dotted lines). The Fermi distribution (red and blue line) specifies that an available strategy $(x)$ will be occupied by the other strategy $\left(x_{f}\right)$ with probability $\left[1+e^{-\beta\left[x-x_{f}\right]}\right]^{-1}$

\section{Part 3: Elementary coordination in circadian rhythm (experimental-based)}

One useful strategy of obtaining simplicity through system dynamic looks for cycles at all time scales (McGhee and Jain 1972) and aims to show how interacting cyclic processes (Collins and Stewart 1993) account for the emergence of new entities (Shaw and Kinsella-Shaw 1988; Turvey and Carello 2012), many of which are similarly cyclic (Yadlapalli et al. 2018). The central idea is that Earth's cycles-geophysical, hydrological, meteorological, geochemical, and biochemical-have interacted to create self-replicating living systems that conform to specific cyclicities (Maury et al. 2010). This assumption has led us to enquire whether something akin to attunement to the environmental 24-h day/night cycles (Maury et al. 2010) may be apparent in an experimental setting of bimanual coordination, a context that has been used to examine self-organization in biological systems (Kugler and Turvey 2015).

The present study used two main ways of determining these characteristics and discovering if approximations under certain conditions serve these self-potentials (see Additional file 1: 2.1 for more detail). The first involves an increase in the capability to self-generate forces along the lines of the roles of the fundamental dimensions of environments (temperature embedding in light-dark cycles). To achieve this, the experimental setting asks, "Is our system influenced by an ecological feature?" by embedding a bimanual coordination task in an ordinary 24-h day-night cycle (5:00, 12:00, 17:00, and 24:00). The second is tied to observing the availability of an internally based source (coordination) or sources of force (stability and entropy) within dynamical boundaries in systematic ways. The setting asks, "How does our system adapt to regular or irregular thermal structures?" by embedding the comparison of normal and abnormal day-night circadian temperature effects at dawn (5 a.m., approximately when the core temperature reaches its minimum) and dusk (5 p.m., 
approximately when the core temperature reaches its maximum) (Aschoff 1983) (see “Methods II (experimental-based model)" for more detail).

\section{Result}

A variety of measures (e.g., phase shift, variability, entropy) were examined for evidence of entrainment or any influence of the embedding rhythm on stability or attractor location (only entropy production was suggested for the main results; see Additional file 1: 2.3 for the entropy calculation). With respect to experiment 1 , the behavioral performance (entropy) shows a maximum at 5:00 but has a more clearly defined minimum at approximately 17:00 in the day-night cycle, while the core body temperature rhythm shows a minimum at 5:00 but has a maximum at approximately 17:00 (see Fig. 3-1).

Regarding experiments 2 and 3, the entropy was affected by the temporal locus during the circadian cycle, as well as by the introduction of a heated vest (Experiment 2) and an ice vest (Experiment 3); the effects of the thermal manipulation were not identical

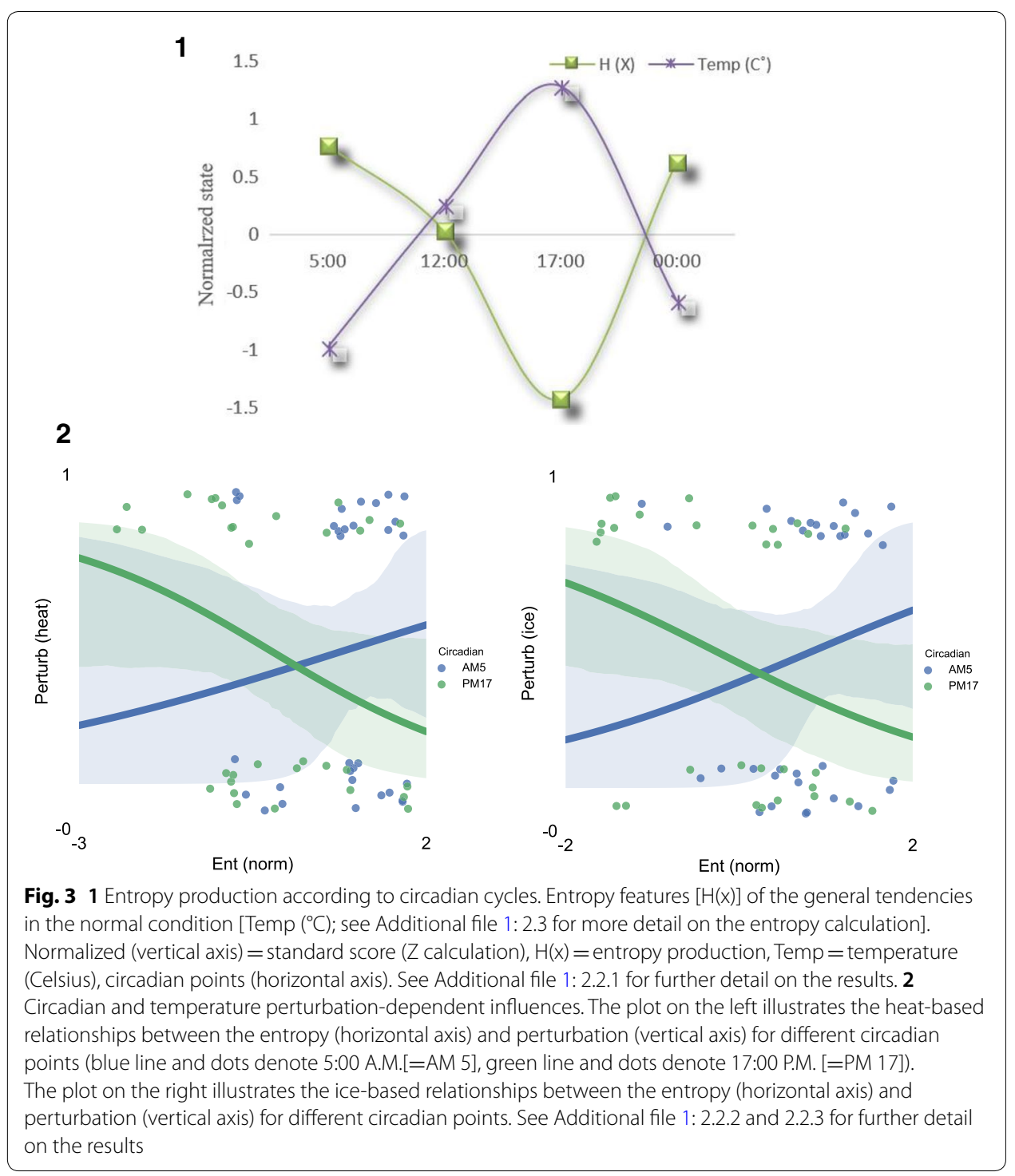


(see Additional file 1: 2.1.4 for the temperature measure). Even if the same external temperature perturbations were given, the influence of the vest was negatively exaggerated (increasing entropy) at dawn but positively exaggerated (decreasing entropy) in the evening (see Additional file 1: 2.2 for more detail).

The estimated dynamics from the relative phase between two limbs, oscillatory coordination, was affected by the temporal locus during the circadian cycle (see Fig. 3-2). The results at this biological scale correspond to a theoretical study that has shown that the rate of entropy production is changed when a new energy source is accessed via a nonequilibrium phase transition process (Frank 2011).

\section{Discussion}

The organism may convert its own internal energy so efficiently that it is able to produce anything physically possible (England 2013). These results from Experiments 1, 2, and 3 reflect that accessing a new energy source differs as a function of the circadian cycle and that access can be manipulated by a temporary thermal manipulation. Given the very widespread use of these features, what types of essential properties are involved in the extended emergent elementary dynamics between oscillators? According to researchers (Pikovsky et al. 2003), the basic element of the coordination $(\phi)$ is equal to $\phi\left(x^{\theta_{1}-\theta_{2}}=\phi\right)$, and such an equation resembles the log base $x$ of, which is equal to $\theta_{1}-\theta_{2}$. Then, with respect to the experimental results, the essential foundation of the symmetry dynamics between oscillators $(\phi)$, the preferred elementary frequency of the individual segment of $x$ to the other relative phase from the intended phase, is nearly equal to the slightly asymmetric potential $\left(x^{\phi_{\text {ave }}-\phi_{0}}=\Delta \omega\right.$ ). Thus, if this logic simply keeps going and the outcome is observed in terms of the approximate relative stability of this coordination dynamic, this logic will have " $x$ to the variation of the relative phase $(h \phi)$ is equal to $\theta_{1}-\theta_{2}=\phi$ multiplied by $\Delta \omega$ ". That is identical to $\left[x^{h \phi}=\phi \cdot(\Delta \omega)\right]$, and this dynamic potential will finally be dependent on $\left[\Delta \omega\left(\mathrm{rad} * x^{-1}\right)\right.$, rad $=$ radian $]$. Pervasive interconnectednesseverything is connected with another thing or other things-suggests that behavior is adapted to perceiving both the nested environmental properties and one's own nested behaviors-a union that organizes actions on surrounding circumstances (Reed 1996). The observation of the direct and robust relationship between biological aspects (body temperature and motor synchrony) and an environmental process (circadian temperature cycle) may echo the adaptation of our system to the environment (Iberall 1977).

\section{Part 4: Approximated common property of the behavioral patterns}

Under these observations, a system may be found to exhibit a variety of hitherto unobserved dynamical behaviors, including cultural evolutionary characteristics and the coexistence of multiple search strategies (Arena et al. 2018). This study proposes that the features investigated are a particularly appropriate assumption in terms of obtaining simplicity from complexity.

$$
h(x)=m\left(s\left(x^{-1}\right)\right), x^{-1} \rightarrow s \stackrel{s\left(x^{-1}\right)}{\longrightarrow} m \stackrel{m\left(s\left(x^{-1}\right)\right)}{\longrightarrow}
$$

The expression $h(x)$ represents our way of modeling that denotes wherever and whenever the evolutionary system is observed. This model takes $(x)$ and inputs $(x)$ into $(s)$ 


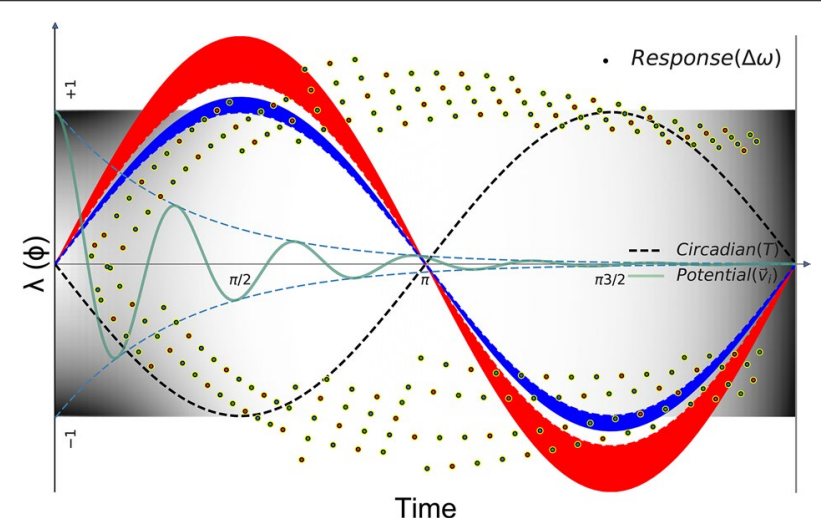

Fig. 4 Schematic illustration of the evolutionary understanding of the behavioral property. The plot represents the state of the system $\lambda(\phi)$ (one arbitrary cycle from - 1.0 to 1.0) over time (horizontal axis). The green line indicates the damping force from model 1 [decay at a maintained value of $\left.d^{2}(\vec{v}) / d t^{2}\right)$ ] between the focal individual and neighbors (or role individual) over time. The contour (black white) represents the 24 -h circadian process as expressed by $[\pi / 2=5: 00, \pi=12: 00, \pi 3 / 2=17: 00$, and (00:00) $]$ according to the optimized value of the system's state with arbitrary units of -1 to 1 . The dotted lines show the observations from model 2 (experimental results). The black line denotes the temperature $(T)$ process according to the circadian cycle. The blue line and shade (distribution) show the observed normal states of the biological system according to the circadian temperature cycle. The red line and shade (distribution) denote the observed abnormal states in the perturbed circadian temperature conditions. The dots surrounded by yellow colors (Response) denote plausible evidence for the association of this property. The crucial variable, which can intuitively be set in both dynamics, can simply be considered as the rate of change between the objects arranged according to a high-sensitivity rule. The results above describe a complex behavior with a divided phase $(\Delta \omega)$ space in which areas of stability are surrounded by confusion. This implies that although their initial states are almost identical (in a comparison of the middle left area of the plot), the response becomes remarkably different with iteration of $n$ times

and obtains $(s(x))$, and then the model inputs that into the $(m)$ and finally takes $m(s(x))$. Going back to the insight by researchers (Mahner and Bunge 1997; Turvey 2018), related to the minimal starting point for understanding any system as a function of interest at any level of interest (see Eq. 1), (s), this includes the collection of all parts of elements $[C(s)]$ and comprises all other things influencing them $[E(s)]$, even the internal structure of the system $[S(s)]$. Thus, we input $(x)$ as arranged as $\left(x^{-1}\right)$ so that it somehow provides us with intuition about a system's fundamental properties $(s)$. This property and the inputs into function $(m)$ help us get to the point of understanding any system based on the system of interest at any level of interest.

As we take this composite function, it models a system that starts with individual segments $(x)$ as the input, and it shows the minimal starting point of the system $(s)$ that will be dependent on the relative individual distance. Thus, how should a system that can be identified or predicted $(h)$ be related to how it depends on the individual segments in a given context? (Melchers and Beck 2018). The fundamental properties demonstrated may be able to create a useful system dynamics reference so that this functional pattern can be applied to various phenomena (see Fig. 4).

Oddly enough, those curiosities might be able to have the same general answer: creation of sophisticated functions from simple elements. There is possible evidence for the association of this property when we compute an approximation of its sensitivity to initial conditions; staying close can show the possible entity as a function of the system's 
own unique set of behavior in the long-time limit $\left[\lambda=\lim _{n \rightarrow \infty}\left(r_{k}^{n}\right)^{1 / n}\right]$. Here, logic can set the object's crucial variable $\left(r_{k}^{n}\right)$ that causes the different value in both dynamics to be considered simply $(1 / n)$ arranged in certain rules (i.e., exponential). This function reflects that the sort of highly sensitive components was associated with a rate of change, which increased or decreased at a certain point (Rosenstein et al. 1993) by measuring the contraction (stable system) or expansion (chaotic system) near the orbit of distance $\left[d\left(x_{0}, x_{0}+\varepsilon\right)\right]$ during the next iteration of distance $\left[d\left(f\left(x_{0}\right), f\left(x_{0}+\varepsilon\right)\right)\right]$ (see Fig. 4 dots surrounded by yellow colors [Response]). That is, the simple rules in regard to what the dynamics provide as the primary characteristics that show the simple function can serve as the basic principle to use when investigating various patterns (Mead 2017).

\section{Conclusion}

One type of logic has focused on the orderly systems underlying the perfect relationships of one point to another (Gaskell and Laughlin 2017). That is to say, there is a direct connection owing to the effect that arises directly from a cause. It follows that if we know the present state of the system, the system can fully determine its past and future state. Although this approach has proven to be a very good means of approximation for describing reality in various fields, the real world in which we actually live is not always covered by these principles (Lear 2012). Most of one's experience might not be due to this type of direct relationship. An important concept for the system, i.e., iterating a simple function, even generates the irregular geometric patterns we see in nature from such a structure (Rian and Asayama 2016). This indicates that there can be an output greater than the sum of the constituting parts due to synergetic relations or less than the sum of its individual components due to the relationships with interference (Haken 2012).

Regarding this assumption behind the simulations which estimated the possible principles of affirmative evolutionary capabilities of a system (Edelman 2016), this study addresses the issues related to the identification of dynamic systems and suggests how determining the basic principles of a collective structure may be key to understanding complex behavioral processes. Distinct approaches (theoretical, modelling, and experimental) were used in an effort to understand how to recognize the basic feature through the dynamics of the system. The condition leading to the behavioral property under these different perspectives suggests that it is not reliant on an individual lodged in the corner of the system. Rather, if the fundamental condition is in place, the dynamics of the system falls into the same rules (Iberall and Soodak 1987) that are estimating the pattern (Rosen 1985). Our validation still needs to be polished against what happens in various fields and then further extending the model, but in general, the approaches and mechanisms achieved here could be useful for researchers and those who need to understand dynamics in phenomena itself.

\section{Methods I (agent-based model)}

The model first considers the agents try to move toward somewhere while steering with other individuals in the group. Thus, each has its position, velocity, and exploration, which are initialized to values randomized within specified continuous space. However, 
at the same time, their pattern of behavior depends not only on the autonomous individuals' initial state but also on its external states. In other words, to control which agents interact, when they interact, and how they interact, the mechanism holds internal traits as a tradeoff (between the individual velocity and group velocity) and external traits as a network characteristic (social ties multiplied by a mutation rate). The rules and processes in the artificially modeled structure describe an individual's homogeneous drives, and the mechanism behind this process is based on the social learning in which successful strategies are propagated through imitation (see Additional file 1: 1.3 for the model variables).

\section{Operating principle}

The agents are physically related to each other on some spatial representation allowing them to move anywhere in the space. The set of $\mathrm{n}$-tuples of a real number, denoted by $\mathbb{R}^{n}$, is called $\mathrm{n}$-spaces $\left[x=\left(x_{1}, x_{2}, \ldots, x_{n}\right) \in \mathbb{R}^{n}\right]$. A particular $\mathrm{n}$-tuples in $\mathbb{R}^{n}$ is a point which called the coordinates, components, or elements of $x$. This is one of the standard ways in which the agents can continue to move in the space. The agents then move within the boundaries of the plane steering toward somewhere.

$$
\vec{u}=\vec{a}_{\text {avg }}+\vec{b}_{i}, \quad \vec{a}_{\text {avg }}=\left\|a_{\text {avg }}\right\| * \vec{d}_{\text {avg }}, \quad \vec{b}_{i}=\left\|b_{i}\right\| * \vec{d}_{i}
$$

where the $\vec{a}$ is the group's heading and $\vec{b}$ is each agent's $(i)$ coherence toward the center of the group. The order $(\vec{u})$ is symmetric because all the agents are identical; thus individuals are naturally heading together in a certain direction, while at the same time maintaining a certain distance from each other as their inherited survival strategies (Jadbabaie et al. 2003).

The model, then, includes another operation with respect to the individual's current movement $(\vec{v})$ written simply by $(\vec{\omega}=\vec{u}+\vec{v})$. That new quantity of $\vec{\omega}$ is the sum of $\vec{u}+\vec{v}$, where the vectors stay away from the origin. The way we define this is that each vector represents a certain movement; a step with a certain distance and direction in space. If we take a step along the first vector of the $\vec{u}$, and then a step in the direction and distance described by the second vector of the $\vec{v}$, the overall effect is just the same as if we had moved along the sum of those two vectors to begin with (see Fig. 5).

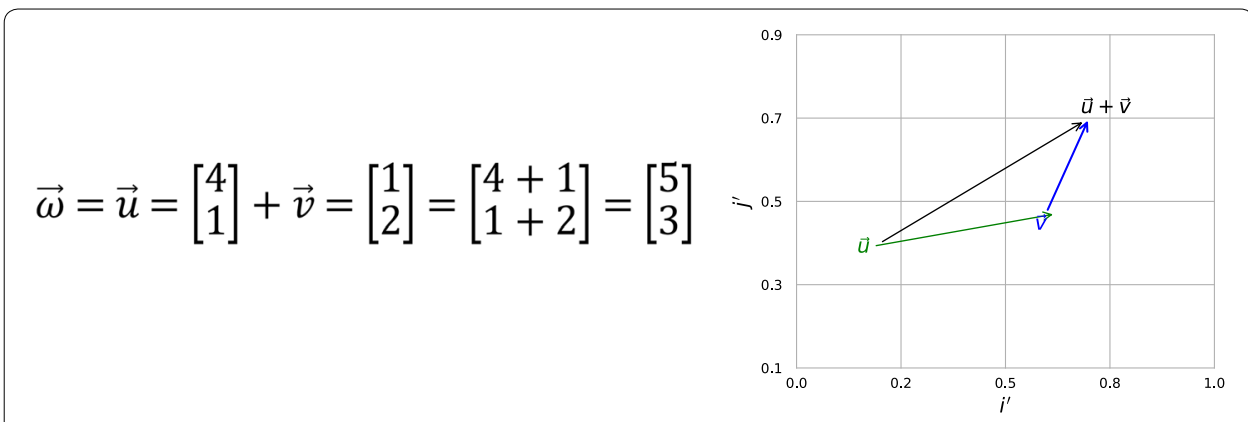

Fig. 5 Schematic representation of the operation [coordinate system: $\hat{i}$ (horizontal axis) and $\widehat{j}$ (vertical axis)] 
The first quantity here (green line) has the coordinate $\left(\vec{u}=\left[\begin{array}{l}4 \\ 1\end{array}\right]\right.$, and the second quantity (blue line) has the coordinate $\left(\vec{v}=\left[\begin{array}{l}1 \\ 2\end{array}\right]\right)$. When we take the sum of the two quantities, we can see a four-step path from the origin to the tip of the second quantity: move 4 to the right and 1 up, then move 1 to the right again and 2 up. To rearrange these steps, first move $4+1$ to the right, then move $1+2$ up; the new quantity (black line) has coordinates $4+1$ and $1+2$ from the origin. Exhibited in this list-of-numbers conception is a matching up of their numerical terms, and an adding them both together $\left(\vec{\omega}=\left[\begin{array}{l}4+1 \\ 1+2\end{array}\right]=\left[\begin{array}{l}5 \\ 3\end{array}\right]\right)$. With this fundamental process, especially, we see that the second quantity of $\vec{v}$ contains the individual's trait (agent-self interactions: agents can interact with themselves), based on its condition holds;

$$
\vec{v} \rightarrow \vec{f} \rightarrow f(\vec{v}), \quad f(\vec{v})=\left\{\begin{array}{l}
\vec{v}(+) \text { if } f\left(\left\|v_{i}\right\|\right)<\left\|v_{\text {avg }}\right\| \\
\vec{v}(-) \text { if } f\left(\left\|v_{i}\right\|\right)>\| v_{\text {avg }}
\end{array}\right.
$$

where the function assumes that the attribute of the component is conditional upon the value yield in the other direction (-), if the length of the magnitude $\left\|v_{i}\right\|$ is greater than the other length $\left(\left\|v_{\text {avg }}\right\|\right)$. This trait is implemented according to quantity as follows:

$$
\vec{v}_{i}=\left\|v_{i}\right\| * \vec{d}_{i}, \quad\left\|v_{i}\right\|=\sqrt{v_{i x}^{2}+v_{i y}^{2}}, \quad \vec{d}_{i}=\frac{\left(v_{i x}, v_{i y}\right)}{\sqrt{v_{i x}^{2}+v_{i y}^{2}}}, \quad \vec{v}_{\text {avg }}=\frac{1}{N} \sum_{i=1}^{N} \vec{v}_{i}=\left\|v_{\text {avg }}\right\| * \vec{d}_{\text {avg }}
$$

where the $\vec{v}_{i}$ is the individual's velocity represented by the length of the individual's magnitude $\left(\left\|v_{i}\right\|\right)$ with the direction of individual $\left(\vec{d}_{i}\right)$. The $\vec{v}_{\text {avg }}$ is their average velocity which includes the entire population of $\mathrm{N}$ individuals' navigation. The result of the $\vec{v}_{i}$ and the $\vec{v}_{\text {avg }}$ produces a new quantity $\vec{v}_{\text {inew }}$ written simply as $\left(\vec{v} \rightarrow \vec{v}_{\text {inew }}=\vec{v}_{i}+\vec{v}_{\text {avg }}\right)$. Going back to the conditional (IF) assumption with this individual quantity, if the object $(\vec{v})$ faces the parameters $(\vec{u})$ with the states of their quantities $\left(\left\|v_{i}\right\|\right.$ and $\left.\left\|v_{\text {avg }}\right\|\right)$, the condition set produces an opposite direction $( \pm)$ depending on its norm as follows:

$$
\begin{aligned}
& f\left(\left\|v_{i}\right\|\right)<\left[v_{\text {avg }}\right] \rightarrow \vec{\omega}=\vec{u}+\vec{v} \\
& f\left(\left\|v_{i}\right\|\right)>\left\|v_{\text {avg }}\right\| \rightarrow \vec{\omega}=\vec{u}+(-\vec{v})
\end{aligned}
$$

where $\vec{\omega}$ is a new position vector of the individual updated by the inherited trait $\vec{u}$ with the individual's current movement $\vec{v}$. This is a linear combination (or inverse transformation $=180^{\circ}$ counterclockwise), something that takes in inputs and spits out an output for each one;

$$
\underbrace{\left[\begin{array}{l}
x_{\text {in }} \\
y_{\text {in }}
\end{array}\right]}_{\text {input }} \rightarrow f \rightarrow \underbrace{\left[\begin{array}{l}
x_{\text {out }} \\
y_{\text {out }}
\end{array}\right]}_{\text {output }}=\underbrace{\left[\begin{array}{ll}
0 & 1 \\
1 & 0
\end{array}\right]}_{\text {matrix }} \underbrace{\left[\begin{array}{l}
x \\
y
\end{array}\right]}_{\text {vector }} \rightarrow f \rightarrow \underbrace{\left[\begin{array}{ll}
0 & 1 \\
1 & 0
\end{array}\right]}_{\text {matrix }} \underbrace{\left[\begin{array}{l}
x \\
y
\end{array}\right]}_{\text {vector }} \text { or } \underbrace{\left[\begin{array}{cc}
0 & -1 \\
-1 & 0
\end{array}\right]}_{\text {matrix }} \underbrace{\left[\begin{array}{l}
x \\
y
\end{array}\right]}_{\text {vector }}
$$




$$
\begin{gathered}
\vec{\omega}=\vec{u}=\left[\begin{array}{l}
4 \\
1
\end{array}\right]+\vec{v}=\left[\begin{array}{l}
1 \\
2
\end{array}\right]=\left[\begin{array}{l}
4+1 \\
1+2
\end{array}\right]=\left[\begin{array}{l}
5 \\
3
\end{array}\right] \\
\vec{\omega}=\vec{u}=\left[\begin{array}{l}
4 \\
1
\end{array}\right]+\vec{v}=\left[\begin{array}{l}
(-) 1 \\
(-) 2
\end{array}\right]=\left[\begin{array}{l}
4+(-) 1 \\
1+(-) 2
\end{array}\right]=\left[\begin{array}{c}
3 \\
-1
\end{array}\right]
\end{gathered}
$$

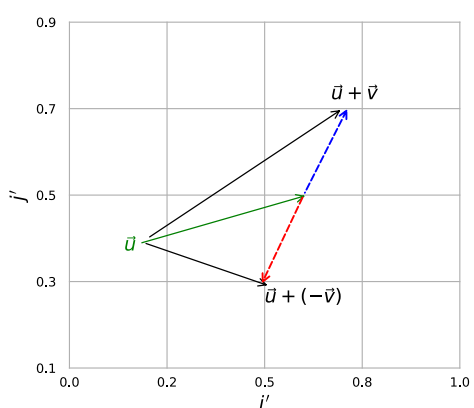

Fig. 6 Schematic representation of the operation [coordinate system: $\hat{i}$ (horizontal axis) and $\hat{j}$ (vertical axis)]

$$
\left[\begin{array}{l}
x_{\text {out }} \\
y_{\text {out }}
\end{array}\right]=\left[\begin{array}{l}
1 \vec{x} \\
1 \vec{y}
\end{array}\right] \text { or }\left[\begin{array}{l}
-1 \vec{x} \\
-1 \vec{y}
\end{array}\right]
$$

Imagine that every possible input vector multiplied by the matrix moves over to its corresponding output vector multiplied by the matrix (or inverse) without becoming curved and that the origin must remain fixed in place; what the coordinates are is determined by where each basis vector lands (see Fig. 6).

This refers to the fact that the model's basic pattern of group behavior depends on the value of $\vec{v}$ with a localized view of the initial conditions of the randomly initialized point; increasing the individual's quantity $\vec{v}$ underlying the group's initial condition $\vec{u}$ causes their portrait to diverge (dotted blue line) or converge (dotted red line). Such a fundamental operation allows us to reach every possible point (not as an arrow but actually as a single point) in the plane, considering every possible linear combination that we can obtain from the two dimensional quantities.

Based on these functions, the element $\vec{v}$ then contains a more detailed algorithm of how the individual's new position was implemented in which a subset holds that (i) the zero vector belongs to $\vec{v}$, and (ii) vectors and any multiplication of scalars is also in $\vec{v}$. Such a mechanism is dependent on quantity as follows.

\section{Movement characteristics (internal source)}

First of all, the new position dynamic is augmented by the designated trade-off value (Woodworth 1899) of the individual velocity-group heading [IGT: individual's velocity up $\left(\vec{v}_{i}\right)$ resulted in a loss of group heading $\left.\left(\vec{v}_{a v g}\right)\right]$ as follows;

$$
\vec{v}_{\text {iinew }}=(1-\|k\|) * \vec{v}_{i}+\|k\| * \vec{v}_{\text {avg }}, \quad k \in[0,1]
$$

where $\vec{v}_{i}$ is the velocity of each individual, $\vec{v}_{\text {avg }}$ is the average velocity about group heading, and the value of $k$ is a scalar that controls their trade-off. For example, the product of a $\vec{v}_{i}$ by a scalar $k$ is a vector $\|k\| \vec{v}_{i}$ with magnitude $\|k\|$ times the magnitude of $\left\|v_{i}\right\|$ and with direction $\vec{d}_{i}$, the same as or opposite to that of $\vec{v}_{i}$, according to whether $k$ is positive or negative [if $k=0$ (null vector) $\|k\| \vec{v}$ has zero magnitude and no specific direction]. This means that every new position vector will be a combination multiplied by the scalar $\left[\left(c_{1}=1-\|k\|\right),\left(c_{2}=\|k\|\right)\right]$ 


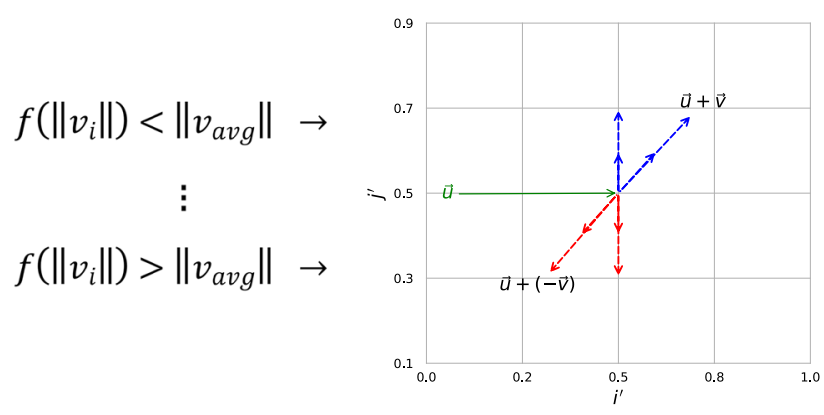

Fig. 7 Schematic representation of the operation [coordinate system: $\widehat{i}$ (horizontal axis) and $\widehat{j}$ (vertical axis)]

$$
\begin{array}{r}
c_{1}\left[\begin{array}{l}
4 \\
1
\end{array}\right] \pm c_{2}\left[\begin{array}{l}
1 \\
2
\end{array}\right]=\left[\begin{array}{l}
c_{1} 4 \\
c_{1} 1
\end{array}\right] \pm\left[\begin{array}{l}
c_{2} 2 \\
c_{2} 2
\end{array}\right]=\left[\begin{array}{l}
x_{1} \\
x_{2}
\end{array}\right] \\
4 * c_{1} \pm 1 * c_{2}=x_{1}, 1 * c_{1} \pm 2 * c_{2}=x_{2}
\end{array}
$$

and, the mechanisms obtain (see Fig. 7):

where the different angles of the arrows are from $\vec{v}_{\text {inew }}$ and the spaces inside the arrows are from the multiplied scalar $(\|k\|,\|k\| \in[0,1])$. This gives us a global view so that we can conceptualize the list of quantities in a visual way and thereby simplify and clarify basic operational patterns.

\section{Network characteristics (external source)}

The model then includes another characteristic for dealing with more or less distinct patterns of behavior. In social practice, just as individuals are more likely to change their decisions depending on the influences surrounding them (Demsar and Bajec 2013), so the individual's new quantity $(\vec{v})$ holds its network characteristics as given;

$$
\vec{v}_{\text {iiinew }}=\left((1-\|k\|) * \vec{v}_{i}+\|k\| * \vec{v}_{\text {avg }}\right)+\vec{v}_{s}, \quad \vec{v}_{s}=\left\|v_{s}\right\| * \vec{d}_{s}
$$

where $\vec{v}_{s}$ is a vector with a length $v_{s}$ and direction $\vec{d}_{s}$ as a function of the network density (ND). The network density arises from its social ties (Santos et al. 2006) based on the nodes and calculated by its actual connection (AC) with the potential connection (PC) of the network.

$$
\left\|v_{s}\right\|=\mathrm{ND}=A C / P C, \quad A C=(2 * t) / N, \quad P C=N(N-1) / 2
$$

where the network density $\left(\left\|v_{s}\right\|\right)$ describes the potential connections in a network that are actual connections $(A C / P C)$. The potential connection $(P C=N(N-1) / 2)$ is a connection that could potentially exist between two individual regardless of whether or not it actually does (see Additional file 1: 1.1 for more detail). These small linear contributions to their dynamics, and this structural instability can be interpreted as the network characteristics being influenced by the exploration rate $\left(k^{\prime}=\right.$ scalar $)$, which corresponds to a mutation term in genetics given as:

$$
\begin{aligned}
& \vec{v}_{\text {iiiinew }}=\left((1-\|k\|) * \vec{v}_{i}+\|k\| * \vec{v}_{\text {avg }}\right)+\vec{v}_{s s}, \vec{v}_{s s}=\left\|v_{s s}\right\| * \vec{d}_{s s} \\
& \left\|v_{s s}\right\|=\left[\left\|k^{\prime}\right\|\left(1-\left\|v_{s}\right\|\right)-2\left\|k^{\prime}\right\|\left\|v_{s}\right\|\right], \quad k^{\prime} \in[0,1]
\end{aligned}
$$




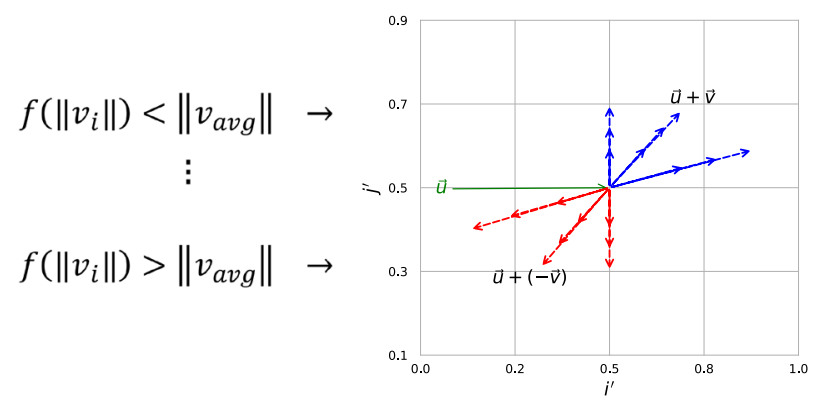

Fig. 8 Schematic representation of the operation [coordinate system: $\widehat{i}$ (horizontal axis) and $\widehat{j}$ (vertical axis)]

where $k^{\prime}$ controls how fast the transition function propagates in the network, and the new position vector considers its network density as another quantity $\left[\left(c_{3}=\left\|k^{\prime}\right\|\right)\right]$. For example, as previously:

$$
\begin{aligned}
& c_{1}\left[\begin{array}{l}
4 \\
1
\end{array}\right] \pm c_{2}\left[\begin{array}{l}
1 \\
2
\end{array}\right]=\left[\begin{array}{l}
c_{1} 4 \\
c_{1} 1
\end{array}\right] \pm\left[\begin{array}{l}
c_{2} 2 \\
c_{2} 2
\end{array}\right]=\left[\begin{array}{l}
x_{1} \\
x_{2}
\end{array}\right] \\
& 4 * c_{1} \pm 1 * c_{2}=x_{1}, \quad 1 * c_{1} \pm 2 * c_{2}=x_{2}
\end{aligned}
$$

The new position is then:

$$
\begin{aligned}
& {\left[\begin{array}{l}
x_{1} \\
x_{2}
\end{array}\right] \pm c_{3}\left[\begin{array}{l}
2 \\
1
\end{array}\right]=\left[\begin{array}{l}
x_{1} \\
x_{2}
\end{array}\right] \pm\left[\begin{array}{l}
c_{3} 2 \\
c_{3} 1
\end{array}\right]=\left[\begin{array}{l}
x_{11} \\
x_{22}
\end{array}\right]} \\
& x_{1} \pm 2 * c_{3}=x_{11}, \quad x_{2} \pm 1 * c_{3}=x_{22}
\end{aligned}
$$

In the presence of the network density, the system settles down into a state with a more pronounced increasing (or decreasing) mutation rate in every update step, and it yields (see Fig. 8):

\section{Interdependency between the two traits}

With these implementations, instead of the widespread extension underlying the combination, the model proposes to adopt an existing possible interconnected relationship between the network and its movement characteristics. Let us think about simple interdependency between the two components (trade-off between individual velocity and group heading as an internal, network density multiplied by mutation as an external). If the individual's tendency is very remote from the group's purpose, its mutation in the system will not propagate to the individuals, or vice versa. For this application, the new position mechanism assumes that the social network characteristics $\left(\left\|v_{s s}\right\|=\right.$ scalar $)$ are a denominator applied by the index of difficulty $(i d=$ scalar $)$ as a numerator.

$$
\begin{aligned}
& \vec{v}_{\text {iiiiinew }}=\left[(1-\|k\|) * \vec{v}_{i}+\|k\| * \vec{v}_{\text {avg }}\right] *\left(\left\|v_{\text {id }}\right\| /\left\|v_{s s}\right\|\right) \\
& \left\|v_{i d}\right\|=\frac{2 D}{W}, \quad\left\|v_{s s}\right\|=\left[\left\|k^{\prime}\right\|\left(1-\left\|v_{s}\right\|\right)-2\left\|k^{\prime}\right\|\left\|v_{s}\right\|\right]
\end{aligned}
$$




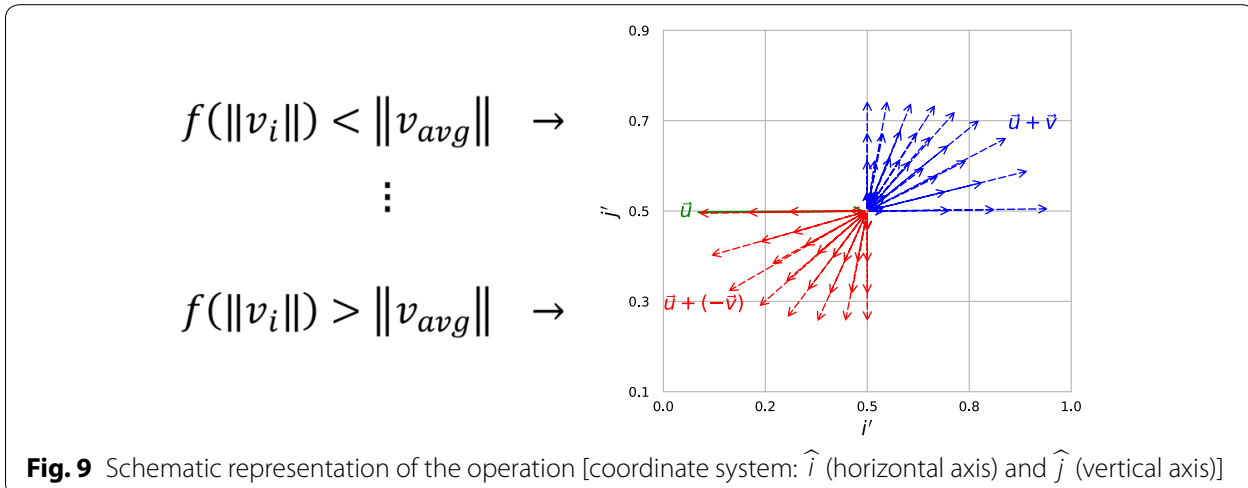

where $\left\|v_{i d}\right\|$ is the scalar as a function of the ratio between the two objects [2D $=$ size of the trade-off $(k)$ between two objects about $\vec{v}_{i}$ and $\vec{v}_{\text {avg }}$ ] divided by the width of the object $[\mathrm{W}=$ arbitrary value corresponding to the individual's size or reputation. For example, when $\|k\|$ is 0.1 applied to the $(1-\|k\|) * \vec{v}_{i}+\|k\| * \vec{v}_{\text {avg }}$, the 2D becomes large (i.e., 0.8 ); on the other hand, when $\|k\|$ is 0.4 , the $2 \mathrm{D}$ becomes small (i.e., 0.2$)]$. This leads to a simple interpretation linking the vector as follows;

$$
c_{4}\left[\begin{array}{l}
x_{1} \\
x_{2}
\end{array}\right]=\left[\begin{array}{l}
c_{4} x_{1} \\
c_{4} x_{2}
\end{array}\right]=\left[\begin{array}{l}
x_{111} \\
x_{222}
\end{array}\right]=\vec{v}_{\text {iiiiinew }}, \quad\left[\begin{array}{l}
x_{1} \\
x_{2}
\end{array}\right]=\vec{v}_{\text {iinew }}, \quad c_{4}=\left\|v_{i d}\right\| /\left\|v_{s s}\right\|
$$

and, the mechanisms yields (see Fig. 9):

Notice that the combination of these arrows refers to this model's fundamental feature. The characteristics of every rule and process that are applied (or will be applied) in this model must be within this functional dynamic. This provides an excellent way of conceptualizing many lists of individuals in a visual way, which can clarify patterns in mechanisms. It also shows a global view of what certain operations do to describe how an individual is being manipulated in space using numbers that can be run through a computation.

The model now considers an adoption probability which is given by an estimate of $\vec{v}_{\text {inew }}$ by the individuals. Indeed, as each individual may not know the exact value of the trait that has adopted the other's $\vec{v}_{\text {inew }}$, this model yields that they can estimate the value at every schedule of each generation via the comparison given.

$$
p=\left[1+e^{-\omega \Delta \pi}\right]^{-1}, \pi_{r}-\pi_{f}=\left.\Delta \pi\right|_{\pi_{r}=\text { rolemodel }}
$$

where $p$ is the probability acceptance of the role model for imitation, $\pi_{f}$ is a payoff (velocity) of the focal individual, $\pi_{r}$ is a payoff of the role individual, $e$ denotes the exponential, and $\omega$ is the intensity of the selection $(\omega<1=$ weak selection, $\omega \rightarrow \infty=$ strong selection). The focal individual imitates the strategy of the nearby role individual, comparing its new position vector (large $\Delta \pi=$ velocity difference large, small $\Delta \pi=$ velocity difference small), and then the focal individual chooses to imitate the strategy of the role individual (see Additional file 1: 1.2 for different implementation cases for the $\pi$ ). 


\section{Methods II (experimental-based model)}

The present model used two main ways of determining these characteristics and discovering whether approximations under certain conditions serve these self-potentials. The first involves an increase in the capability to self-generate forces along the lines of the roles of the fundamental dimensions of environments (temperature embedding in light-dark cycles). To achieve this, the experimental setting asks, "Is our system influenced by an ecological feature?" by embedding a bimanual coordination task in an ordinary 24-h day-night cycle (5:00, 12:00, 17:00, and 24:00). The second is tied to observing the availability of an internally based source (coordination) or sources of force (stability and entropy) within dynamical boundaries in systematic ways. The setting asks, "How does our system adapt to regular or irregular thermal structures?" by embedding the comparison of normal and abnormal day-night circadian temperature effects at dawn (5 a.m., approximately when the core temperature reaches its minimum) and dusk ( 5 p.m., approximately when the core temperature reaches its maximum) (Aschoff 1983) (see Additional file 1: 2.1 for more detail).

\section{Circadian rhythm of temperature (external source)}

The core cycles of a biological system are influenced by temperature, with 24-h lightdark oscillation (called circadian rhythm), as well as by biochemical, physiological, or behavioral processes that persist under constant conditions with a period length of $\sim 24$ (Soodak and Iberall 1978). Presumably, due to inputs to the thermoregulatory centers from the body core (Refinetti and Menaker 1992), the circadian rhythm of biology shows a minimum at 5:00 (when core body temperature is rising most rapidly) but has a more clearly defined maximum at about 17:00 in the daylight (when core body temperature is falling most rapidly) cycle (Moore 1995) (see Fig. 10).

This circadian change (in core temperature) is most likely due to the rhythmic input from the suprachiasmatic nuclei $(\mathrm{SCN})$ acting upon the hypothalamic thermoregulatory centers and altering the thresholds of cutaneous vasodilatation and sweating (Krauchi and Wirz-Justice 1994). Specifically, melatonin appears to contribute to this change, as its rate of secretion increase in the evening, and this increase promotes a fall in body temperature via cutaneous vasodilatation (Cagnacci et al. 1997).

As the information is accessible, people are familiar with how such a process can fluctuate and how it can be explained by the interaction between the internal (homeostatic) and the external (circadian) situations (Aizawa and Cabanac 2002). There is ample evidence of the effect of the ecological climate on various aspects of the process. Heat

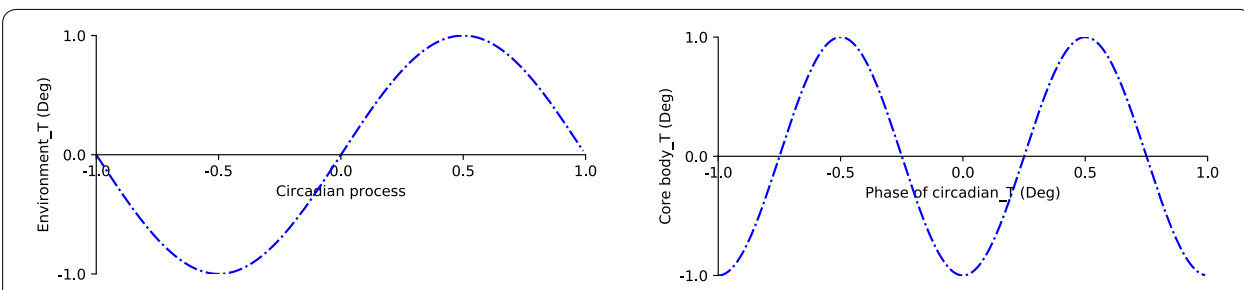

Fig. 10 Representation of the circadian rhythm. Left = circadian process oscillation; right = temperature process oscillation between the circadian temperature (horizontal axis) and the body temperature (vertical axis). Note: this is a normalized rhythm despite the fact not all rhythms are identical. Our core body temperature is roughly linked to this cycle, with various hormones being released at certain stages during the rhythm because our body temperature reflects energy levels 
exchanged with the environment by means of convection and radiation allows a gradient to be formed between the body core and temperature (Borb and Achermann 1999; Walther et al. 2002). The rhythm in the core temperature produced by this change is generally promoted by other rhythms, including the body clock, sleep, and physical and mental activity, raising the possibility that the disruption of circadian rhythms can contribute to complications in the human system (Maury et al. 2010). These changes in the interior temperature in the body, as opposed to the peripheral (core temperature)-both in animals and humans-are mainly due to circadian rhythmic changes in the rates of ecological impacts (Edholm et al. 1973).

However, given that precise control of the internal substance $(\mathrm{SCN})$ as a generator of biological circadian rhythm is unclear, the circadian rhythm of the core body temperature appears to be generated by periodic variation in heat production and heat loss (Waterhouse et al. 2005). For instance, changes in heat loss via convection and radiation are primarily caused by variations in skin blood flow, with consequent changes in skin temperature. In particular, when the subject is performing mild activities, where a decreased temperature is not matched by a thermal load, it has been shown to be very effective in describing the thermal responses to activity carried out at different times of the day (Aschoff et al. 1972).

When one considers the submaximal activity changes following a brief period, say, at a certain temperature level (Aldemir et al. 2000), one may see initially that the response to the same amount of moderate activity in the minimum circadian rhythm differed from that in the maximum circadian rhythm. The mechanisms responsible for these different temperatures of the core and musculature during daylight cycles, as a result of normal or non-normal ambient temperatures, will alter a range of performance factors, including the thermoregulatory response to activity. These results fully substantiate the predictions based on the hypothesis describing a circadian rhythm in thermoregulatory responses and indicate that this hypothesis applies to biological adaptation regarding certain ecological variables.

\section{Elementary coordination (internal source)}

Formation and retention refer to propriospecific information about the states of the muscular-articular links, and the dynamical criteria of the stability pattern constrain the patterns or characteristics. To be specific, let us consider a qualitative physical system such as stiffness, damping, and position over time in a dynamical mass-spring system as given.

$$
\mathrm{f}(\mathrm{t})=m x^{\prime \prime}+b x^{\prime}+k x
$$

Here, $m$ is mass, $b$ is friction, and $k$ denotes the stiffness. The variable $\mathrm{t}$ is time, $\chi$ denotes the position, $\chi^{\prime}$ is velocity, and $\chi^{\prime \prime}$ represents acceleration. In physics, because damping is produced by a process that dissipates the energy stored in the oscillations, the interplay between input and damping approaches a stationary fixed point in the long-time limit.

$$
m x^{\prime \prime}+b x^{\prime}+k x=0
$$




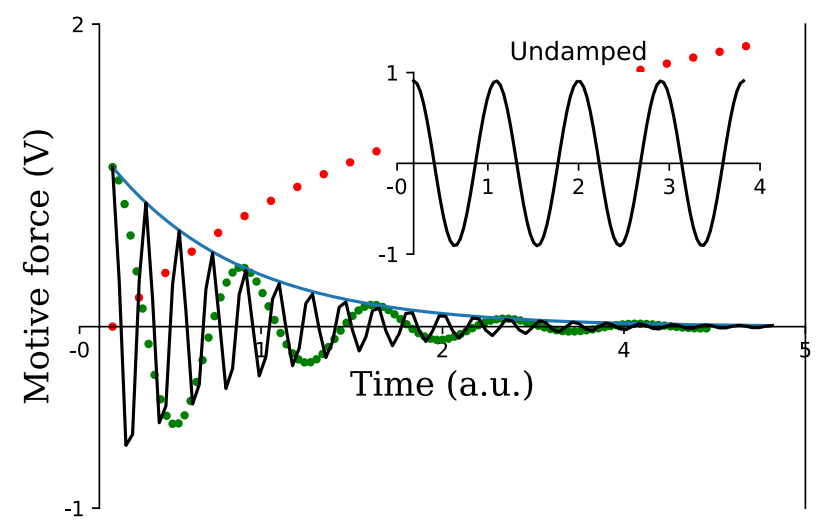

Fig. 11 Simulation of the different mass-spring attractors. The damped exponential decay of the dotted equals $\cos (2 \pi t) \exp (-t)$, and for the solid line $\cos (8 \pi t) \exp (-t)$. Log lines indicate an embedded invariant property in terms of the relation between systems' attractor (solid blue line) and damping potential (dotted red line). $V$ volts, au arbitrary unit. Plot of the inset denotes an undamped case

Such systems possess a static equilibrium point, which is called a point attractor (Kugler et al. 1980) (see Fig. 11). The property of this dynamic has been applied not only to a physical system but also to descriptions of the human neuromuscular level (Kugler and Turvey 2015). This function involves an investigation of the intact movement of a limb oscillator in terms of muscle-joint kinematic variations (kinematic position, velocity, acceleration) over time. When we are asked to swing two limbs comfortably, this can be characterized by the pendulum's dimension (Kay et al. 1987), namely, simplifying the point attractor while restricting it to certain domains of phase space $[(\theta 2-\theta 1 \approx 0),(\theta 2-\theta 1 \approx \pi)]$. In this equation, with the phase difference, $\theta 2-\theta 1 \approx 0$ denotes a condition of nearly synchronized in-phase, and $\theta 2-\theta 1 \approx \pi$ indicates that this in an anti-phase. The observed relative phase or phase relation $(\phi)$ between two oscillators at $\phi \approx 0^{\circ}$ (in-phase), or $\phi \approx 180^{\circ}$ (anti-phase) have been modeled as the point attractors in our limb system, as they are purely stable patterns (Turvey 1990).

In the observed relative rhythmic segments patterns, the in-phase $\phi \approx 0$ condition is more stable than the anti-phase $\phi \approx \pi$ condition. Inspired by a number of studies on the 1:1 frequency locking of the left- and right-hand phase defined as $\phi=\left(\theta_{L}-\theta_{R}\right)$ -the difference between the left $(\mathrm{L})$ and right $(\mathrm{R})$ phase angles $(\phi)$-has led to the identification of important invariant human system features (Kelso 1984).

$$
\mathrm{V}(\phi)=-\alpha \cos (\phi)-\mathrm{b} \cos (2 \phi)
$$

In this equation, $\phi$ is the phase angle of the individual oscillator. In addition, $\alpha$ and $\mathrm{b}$ are coefficients that denote the strength of the coupling between the two oscillators. The relevant regions of the parameter space allow the potential $\mathrm{V}(\phi)$; the negative signs in front of the coefficients simplify the equation of motion. A relative 1:1 frequency-locked coordination phase $[\mathrm{V}(\phi)]$ is determined by the differences between the continuous phase angle $[-\alpha \cos (\phi)-b \cos (2 \phi)]$ of the oscillator's two components: the stability of the point attractor can be varied by varying the pendulum's dimensions (see Fig. 12). 


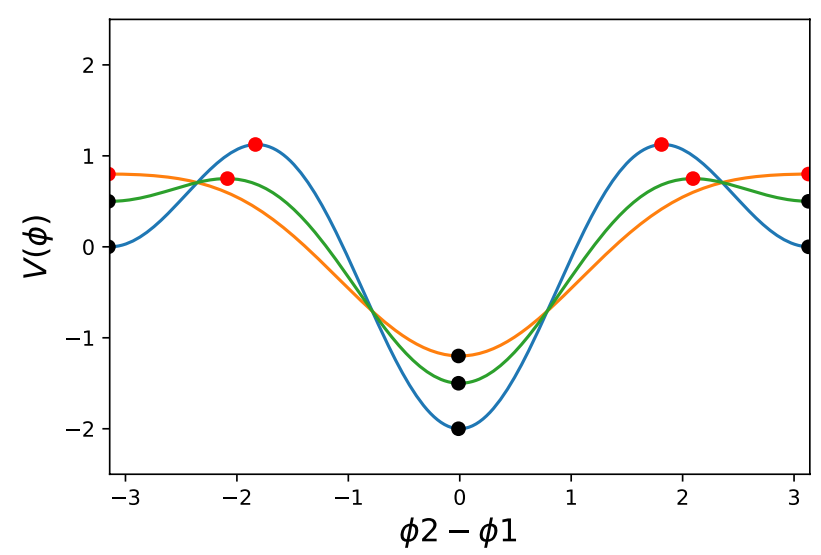

Fig. 12 Reflection of a potential function. The blue line = the vertical axis, which denotes the energy of the function at each averaged relative phase. The horizontal axis indicates the averaged relative phase between two limbs from in-phase 0 to anti-phase $3.14=180(-3.14=-180)$. At the local point 0 and $180(-180)$, the function close to those minima (attractors, black balls) and at local point around $90(-90)$, the state is close to maxima (repellors, red balls). The red and green lines denote the variation of the potential functions

This function indicates that the minima of the potential are located at $\phi=0$, and that $\phi= \pm \pi$ (Haken et al. 1985). Given this scenario, the function can be estimated in terms of how the potential will change in shape, as the control parameter (energy cost) increases. Based on the observed mechanism for the point attractor with a simple function, the present model proposes the in-phase bimanual rhythmic coordination synchrony pattern as a particularly well-suited physical source. This allows a useful reference for system stability coordination tasks in which this functional pattern can be applied to all human movement, muscles, and even a neural network. Actual intersegmental coordination, however, is additionally shaped by the contingencies of adjusting to environmental vagaries. How these extrospecific requirements and information types are incorporated into the physical stability patterns can be assumed by the level of symmetry coordination (Amazeen et al. 1998). In order to harmonize the effects of motor stability toward environmental symmetry, this study investigates the following elaboration.

\section{Symmetry breaking in bimanual coordination dynamics}

The potential $[\mathrm{V}(\phi)]$ extends the described assumption in terms of the difference between the uncoupled frequencies of bimanual rhythmic components $\left[\Delta \omega=\left(\omega_{L}-\omega_{R}\right)\right]$. Where $\omega$ is the preferred movement frequency of the left $\left(\omega_{L}\right)$, right $\left(\omega_{R}\right)$ individual. If the relative phase between $\omega_{L}$ and $\omega_{R}$ were equal $(\Delta \omega=0)$, this pattern would be assumed to be a perfectly identical symmetry. However, the preferred movement frequencies of the individual oscillators in in-phase are large (i.e., function: $\mathrm{b} / \mathrm{a}=0.5$, detuning $=-0.5$, or detuning $=-1.5$ ), the expected stability of the rhythmical limb oscillation dynamics become greater than equal (see Fig. 13).

Such phenomena of the symmetry breaking must be another fundamental feature of the coordinative system (Amazeen et al. 1998). From this dynamic, a different noise of the underlying subsystems (neural, muscular, and vascular) can be estimated around 


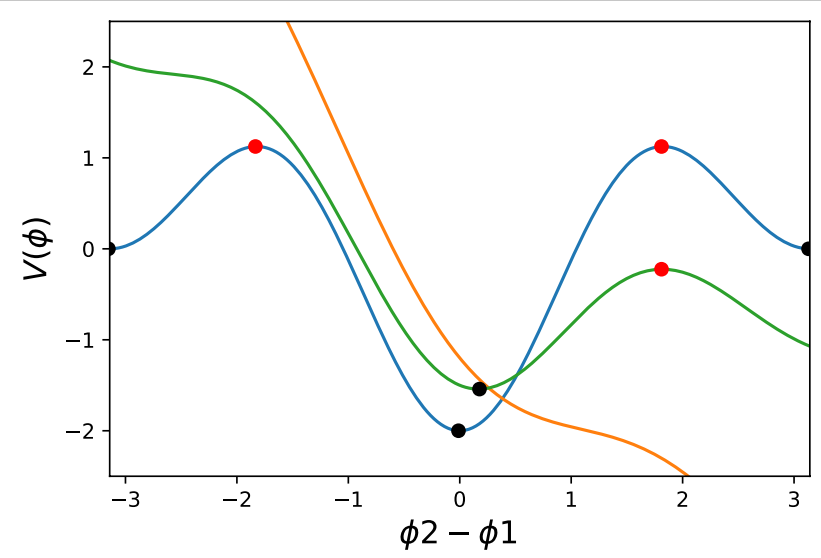

Fig. 13 Preferred movement frequencies of the individual oscillators. The vertical axis, which denotes the energy of the function at each averaged relative phase. The horizontal axis indicates the averaged relative phase between two limbs from in-phase 0 to anti-phase $3.14=180(-3.14=-180)$. The blue line denotes the same symmetry; green line denotes the large different symmetry (function: $b / a=0.5$, detuning $=-0.5$ ); redline denotes even larger different symmetry (function: $\mathrm{b} / \mathrm{a}=0.5$, detuning $=-1.5$ )

an equilibrium point, and this might conceptualize the model when it comes to making operational definitions of each category in which the model has to consider the variability of the relative phase frequencies between two limbs:

$$
\dot{\phi}=\Delta \omega-\alpha \cos (\phi)-\mathrm{b} \cos (2 \phi)+\sqrt{\varrho \xi_{t}}
$$

The estimation of two oscillators' relative phase $(\dot{\phi})$ is captured by the parameter $(\Delta \omega)$ of the preferred movement frequency of the individual segment $[\alpha \cos (\phi)-b \cos (2 \phi)]$ with the noise $\left(\sqrt{\varrho \xi_{t}}\right)$. Given the equation of the preceding model (grouped as the kinematics of motor stability according to the coordination task of synchronization), such a term has been used to capture purely functional dynamics regarding the equilibria and is confirmed usually as in the time and temporal difference between an oscillating limb.

Researchers (Treffner and Turvey 1995), conducting experiments in handedness, advanced the elementary coordination dynamics. They added two add (sine) terms for the coefficients, whose signs and magnitudes determine the degree and direction of asymmetry, as follows;

$$
\dot{\phi}=\Delta \omega-[\alpha \sin (\phi)+2 b \sin (2 \phi)]-[c \sin (\phi)+2 d \sin (2 \phi)]+\sqrt{\varrho \xi_{t}}
$$

Here, $\dot{\phi}$ indicates a coordination change. $\Delta \omega$ refers to a symmetry breaking through frequency competition between two limbs. $[\alpha \sin (\phi)+2 b \sin (2 \phi)]$ denotes a symmetric coupling defined by relative phase of 0 and $\pi$ attractors (this form of the term could be derived as the negative gradient potential $\mathrm{V}$ with respect to $\phi$ ); and the $[c \sin (\phi)+2 d \sin (2 \phi)]$ terms means added asymmetric coupling attractors with the stochastic noise $\sqrt{\varrho \xi_{t}}$. This extended equation refers to the fact that the emergent elementary dynamics between limbs or limb segments was governed by a slightly asymmetric potential of the $[c \sin (\phi)+2 d \sin (2 \phi)]$. That suggests extended collective dynamics of the inter-segmental rhythmic coordination of the periodic components. 


\section{Thermoregulatory symmetry breaking of the elementary coordination}

Inspired by the complementary symmetric and asymmetric influences, the described model was applied to investigate the difference between the coupled or uncoupled frequencies of the temperature-rhythmic components between the core body and circadian cycles.

$$
c=\text { circadian temperature cycle }, d=\text { core body temperature cycle. }
$$

where $d$ is the preferred rhythmic frequency of one (the homeostasis cycle) and another ( $c=$ circadian cycle) individual. Whereas $\mathrm{b} / \mathrm{a}$ determines the relative strengths of the fundamental in-phase equilibria, small values of $c$ and $d$ break the symmetry of the elementary coordination dynamics while leaving their essential coupling characteristics.

$$
\mid c \text { and } d|>0, \quad| \text { and } d \mid \approx 0
$$

In this proposed assumption, the coefficient of the $d$ should be more important, producing the empirically observed perturbation in the equilibrium phase state, and then the $c$ should be set to zero without loss of generality, given that we cannot manipulate the environmental circadian cycle. As one can see, if the coupling between $d$ and $c$ is strong $(\mid c$ and $d \mid \approx 0$ ), this pattern would potentially be expected to be in perfectly corresponding symmetry with the environmental requirement. However, the preferred rhythmic coupling of individual oscillators in an in-phase condition becomes a difference $(\mid c$ and $d \mid>0)$, and thus the expected stability or variability of the rhythmical-component oscillation dynamics will become greater than equal. Given the preceding assumption (grouped as the kinematics of motor stability according to the coordination task of synchronization), the equation was extended to a novel task in which there are different sources of symmetry breaking through thermal variables, as information has not yet been made available about the effects of bimanual dynamics in instruction on circadian temperatures.

$$
\dot{\phi}=\Delta \omega-[\alpha \sin (\phi)+2 b \sin (2 \phi)]-\left[c \sin \left(\phi^{\circ} \mathrm{C}\right)+2 d \sin \left(2 \phi^{\circ} \mathrm{C}\right)\right]+\sqrt{\varrho \xi_{t}}
$$

In this equation, in the bimanual 1:1 rhythmic coordination performed at different coupled frequencies, the symmetric coupling coefficients will be not the same. There will be an increase in detuning $(\Delta \omega)$ and a decrease in the relative strengths of the attractors at 0 and $\pi$. However, when it comes to our limiting case of $\Delta \omega=0$ on the approximately identical symmetry temperature parameters (core body and circadian cycle), what should we expect? The final estimation between the relative phases of two oscillators $(\dot{\phi})$ will be captured mainly by the parameter of the asymmetric thermoregulatory coupling $\left[c \sin \left(\phi^{\circ} \mathrm{C}\right)+2 d \sin \left(2 \phi^{\circ} \mathrm{C}\right)\right]$ with noise $\left(\sqrt{\varrho \xi_{t}}\right)$. From this dynamic, the different noise types of the underlying subsystems (neural, muscular, and vascular) around an equilibrium point were able to be estimated, suggesting that such phenomena related to symmetry breaking may be yet another remarkable feature of the coordinative system.

In sum, this experiment was required to have a condition of in-phase $(\phi=0)$ oscillated simultaneously at the 1:1 frequency locking (the in-phase was suggested for the 
main variable based on the pilot test; see Additional file 1: 2.4 and 2.5 for more detail). The same goal using the functional symmetry dynamics of different effectors will be influenced by the asymmetric thermal regulation symmetry breaking through both circadian temperature cycles. Namely, the effect of one of the contralateral homologous relative limbs phase might be not identical to the impact of the others. The expected stability pattern, from intuition given a different motor, appears to allow the biological symmetry dynamic to be understood in the ecological context. This attunement to the circadian temperature approach implies an emergent property of the system.

\section{Supplementary information}

Supplementary information accompanies this paper at https://doi.org/10.1186/s40294-019-0066-X.

Additional file 1. 1. Individual behavior in social dynamics (Model 1); 1.1 Applied network structure, 1.2 Three implementations for Pi, 1.3 Table of the model variables, 1.4 Results supplements. 2. Elementary coordination in circadian cycles (Model 2); 2.1 Experimental designs, 2.2 Results supplements, 2.3 Entropy calculation, 2.4 Preliminary pilot test for the experiments, 2.5 Graphical illustrations of the inclusion andexclusion case of the data.

\section{Acknowledgements}

The author sincerely thanks the experimental participants at University of Connecticut and Seoul National University for their patience. All of them provided written informed consent to the study approved by the local ethics committee (SNUIRB No. 1509/002-002) and conformed to the ethical standards of the 1964 Declaration of Helsinki (report ID 20481572). The authors would also especially wish to thank Seonjin Kim at Laboratory of Motor Behavior (Seoul, Korea), Hyeongsaeng Park at Cognitive Science Program (Seoul, Korea), Claudia Carello at Center for the Ecological Study of Perception and Action (Connecticut, USA), Ulf Dieckmann at International Institute for Applied Systems Analysis (Laxenburg, Austria) for their fruitful discussion and the valuable support during the development of this article.

\section{Authors' contributions}

CP designed the model, did the analysis and tested it, and wrote the paper. The author read and approved the final manuscript.

\section{Funding}

This research was supported by an NSF Grant BCS-1344725 (INSPIRE Track 1), and the National Research Foundation of Korea (2016K2A9A1A02952017).

\section{Availability of data and materials}

All data and materials are our own. The materials and data used to support findings of this study are included within the additional file.

\section{Competing interests}

The author declares no competing interests.

\section{Author details}

${ }^{1}$ International Institute for Applied Systems Analysis (IIASA), 2361 Laxenburg, Austria. ${ }^{2}$ Department of Physical Science, Seoul National University Institute of Sport Science, 08826 Seoul, South Korea.

Received: 5 October 2019 Accepted: 9 December 2019

Published online: 12 February 2020

\section{References}

Aizawa S, Cabanac M (2002) The influence of temporary semi-supine and supine postures on temperature regulation in humans. JTherm Biol 27(2):109-114

Aldemir H, Atkinson G, Cable T, Edwards B, Waterhouse J, Reilly T (2000) A comparison of the immediate effects of moderate exercise in the early morning and late afternoon on core temperature and cutaneous thermoregulatory mechanisms. Chronobiol Int 17(2):197-207

Amazeen PG, Amazeen EL, Turvey MT (1998) Breaking the reflectional symmetry of interlimb coordination dynamics. J Mot Behav 30(3):199-216

Arena M, Azzone G, Mapelli F (2018) What drives the evolution of corporate social responsibility strategies? An institutional logics perspective. J Clean Prod 171:345-355

Aschoff J (1983) Circadian control of body temperature. J Therm Biol 8:143-147

Aschoff J, Heise A, Itoh S, Ogata K, Yoshimura H (1972) Advances in climatic physiology. Igaku Shoin 334:348

Ashby W (1947) Principles of the self-organizing dynamic system. J Gen Psychol 37:125-128

Barabasi AL (2005) The origin of bursts and heavy tails in human dynamics. Nature 435:207-211

Bernstein N (1966) The co-ordination and regulation of movements. Pergamon Press, Oxford 
Bonabeau E (2002) Agent-based modeling: methods and techniques for simulating human systems. Proc Natl Acad Sci USA 99:7280-7287

Borb AA, Achermann P (1999) Sleep homeostasis and models of sleep regulation. J Biol Rhythms 14(6):559-570

Borgonovi F, Izrailev FM, Santos LF (2019) Exponentially fast dynamics of chaotic many-body systems. Phys Rev E 99:010101

Cagnacci A, Kräuchi K, Wirz-Justice A, Volpe A (1997) Homeostatic versus circadian effects of melatonin on core body temperature in humans. J Biol Rhythms 12(6):509-517

Chen S, Billings S (1992) Neural networks for nonlinear dynamic system modelling and identification. Int J Control 56:319-346

Collins JJ, Stewart IN (1993) Coupled nonlinear oscillators and the symmetries of animal gaits. J Nonlinear Sci. 3:349-392

Demsar J, Bajec I (2013 ) Family bird: a heterogeneous simulated flock. In: Artificial life conference proceedings 13. One Rogers Street, Cambridge, 02142-1209 USA journals-info@ mit.edu: MIT Press, pp. 1114-1115

Edelman S (2016) The minority report: some common assumptions to reconsider in the modelling of the brain and behaviour. J Exp Theor Artif Intell 28:751-776

Edholm OG, Fox RH, Wolf HS (1973) Body temperature during exercise and rest in cold and hot climates. Arch Sci Physiol 27(3):339

England JL (2013) Statistical physics of self-replication. J Chem Phys 139:09B623_1

Ford BJ (2008) Microscopical substantiation of intelligence in living cells. Infocus Magazine. 12:6-12

Frank T (2011) Collective behavior of biophysical systems with thermodynamic feedback loops: a case study for a nonlinear Markov model — the Takatsuji system. Mod Phys Lett B 25:551-568

Gaskell DR, Laughlin DE (2017) Introduction to the thermodynamics of materials. CRC Press, Boca Raton

Gibson J (1979) The ecological approach to visual perception. Houghton Mifflin, Boston

Gigerenzer G (2004) Fast and frugal heuristics: the tools of bounded rationality. In: Koehler D, Harvey N (eds) Blackwell handbook of judgment and decision making. Blackwell, Oxford, pp 62-88

Haken $\mathrm{H}$ (2012) Advanced synergetics: instability hierarchies of self-organizing systems and devices. Springer, Berlin

Haken H, Kelso JS, Bunz H (1985) A theoretical model of phase transitions in human hand movements. Biol Cybern 51(5):347-356

Iberall AS (1977) A field and circuit thermodynamics for integrative physiology. I. Introduction to the general notions. Am J Physiol 233:R171-R180

Iberall A, Soodak H (1987) A physics for complex systems. In: Yates F (ed) Self-organizing systems: the emergence of order. Plenum Press, New York, pp 499-520

Jadbabaie A, Lin J, Morse AS (2003) Coordination of groups of mobile autonomous agents using nearest neighbor rules. Departmental Papers (ESE). p. 29

Kay BA, Kelso JA, Saltzman EL, Schöner G (1987) Space-time behavior of single and bimanual rhythmical movements: data and limit cycle model. J Exp Psychol Hum Percept Perform 13(2):178

Kelso JA (1984) Phase transitions and critical behavior in human bimanual coordination. Am J Physiol Reg Integr Comparat Physiol 246(6):R1000-R1004

Krauchi K, Wirz-Justice AN (1994) Circadian rhythm of heat production, heart rate, and skin and core temperature under unmasking conditions in men. Am J Physiol Reg Integr Comparat Physiol 267(3):R819-R829

Kugler PN, Turvey MT (2015) Information, natural law, and the self-assembly of rhythmic movement. Routledge, New York

Kugler PN, Kelso JS, Turvey MT (1980) On the concept of coordinative structures as dissipative structures: I. Theoretical lines of convergence. Adv Psychol 1:3-47

Lear CW (2012) The Bell inequality and nonlocal causality. J Sci Explore 26:767-779

Mahner M, Bunge M (1997) Foundations of biophilosophy. Springer, New York

Mandl CE (2019) Managing complexity in social systems: leverage points for policy and strategy. Springer International Publishing, Cham

Marchal J (1975) On the concept of a system. Philos Sci. 42:448-468

Maury E, Ramsey KM, Bass J (2010) Circadian rhythms and metabolic syndrome: from experimental genetics to human disease. Circ Res 106:447-462

McGhee RB, Jain AK (1972) Some properties of regularly realizable gait matrices. Math Biosci 13:179-193

McLeod P, Dienes Z (1996) Do fielders know where to go to catch the ball or only how to get there? J Exp Psychol Hum Percept Perform 22:531

Mead R (2017) Statistical methods in agriculture and experimental biology. Chapman and Hall/CRC, London

Melchers RE, Beck AT (2018) Structural reliability analysis and prediction. Wiley, Hoboken

Moore RY (1995) Organization of the mammalian circadian system. In: Ciba foundation symposium. Wiley, New York, p. 88

Oliva R (2016) Structural dominance analysis of large and stochastic models. Syst Dyn Rev 32:26-51

Pattee HH (1973) The physical basis and origin of hierarchical control. In: Pattee HH (ed) Hierarchy theory. G. Braziller, New York, pp 73-108

Pikovsky A, Kurths J, Rosenblum M, Kurths J (2003) Synchronization: a universal concept in nonlinear sciences. Cambridge University Press, Cambridge

Rangel TF, Edwards NR, Holden PB, Diniz-Filho JAF, Gosling WD, Coelho MTP et al (2018) Modeling the ecology and evolution of biodiversity: biogeographical cradles, museums, and graves. Science 361 :eaar5452

Reed ES (1996) Encountering the world: toward an ecological psychology. Oxford University Press, New York

Reed N, McLeod P, Dienes Z (2010) Implicit knowledge and motor skill: what people who know how to catch don't know. Conscious Cogn 19:63-76

Refinetti R, Menaker M (1992) The circadian rhythm of body temperature. Physiol Behav 51(3):613-637

Reynolds CW (1987) Flocks, herds and schools: a distributed behavioral model. ACM Comput Graph 21:25-34

Rian IM, Asayama S (2016) Computational design of a nature-inspired architectural structure using the concepts of selfsimilar and random fractals. Autom Constr 66:43-58 
Rosen R (1985) Anticipatory systems: philosophical, mathematical, and methodological foundations. International federation for systems research. Pergamon Press, Oxford

Rosen R (1987) On complex systems. Eur J Oper Res 30:129-134

Rosen R (1991) Life itself: a comprehensive inquiry into the nature, origin, and fabrication of life. Columbia University Press, New York

Rosenstein MT, Collins JJ, De Luca CJ (1993) A practical method for calculating largest Lyapunov exponents from small data sets. Physica D 65(1-2):117-134

Roundy PT, Bradshaw M, Brockman BK (2018) The emergence of entrepreneurial ecosystems: a complex adaptive systems approach. J Bus Res 86:1-10

Santos FC, Pacheco JM, Lenaerts T (2006) Cooperation prevails when individuals adjust their social ties. PLoS Comput Biol 2(10):e140

Schöner G (2002) Dynamical systems approaches to neural systems and behavior. In: Smelser N, Baltes P (eds) International encyclopedia of the social \& behavioral sciences. Pergamon, Oxford, pp 10571-10575

Shaw R (2001) Processes, acts, and experiences: three stances on the problem of intentionality. Ecol Psychol 13:275-314

Shaw R, Kinsella-Shaw J (1988) Ecological mechanics: a physical geometry for intentional constraints. Hum Mov Sci 7:155-200

Soodak H, Iberall A (1978) Homeokinetics: a physical science for complex systems. Science 201 (4356):579-582

Strong D Jr, Ray T Jr (1975) Host tree location behavior of a tropical vine (Monstera gigantea) by skototropism. Science 190:804-806

Treffner PJ, Turvey MT (1995) Handedness and the asymmetric dynamics of bimanual rhythmic coordination. J Exp Psychol Hum Percept Perform 21(2):318

Turvey MT (1990) Coordination. Am Psychol 45(8):938

Turvey MT (2018) Lectures on perception: an ecological perspective. Routledge, New York

Turvey MT, Carello C (2012) On intelligence from first principles: guidelines for inquiry into the hypothesis of physical intelligence (PI). Ecol Psychol 24:3-32

Walther GR, Post E, Convey P, Menzel A, Parmesan C, Beebee TJ, Fromentin JM, Hoegh-Guldberg O, Bairlein F (2002) Ecological responses to recent climate change. Nature 416(6879):389

Waterhouse J, Drust B, Weinert D, Edwards B, Gregson W, Atkinson G, Kao S, Aizawa S, Reilly T (2005) The circadian rhythm of core temperature: origin and some implications for exercise performance. Chronobiol Int 22(2):207-225

Woodworth RS (1899) Accuracy of voluntary movement. Psychol Rev 3(3):i

Yadlapalli S, Jiang C, Bahle A, Reddy P, Meyhofer E, Shafer OT (2018) Circadian clock neurons constantly monitor environmental temperature to set sleep timing. Nature 555:98-102

\section{Publisher's Note}

Springer Nature remains neutral with regard to jurisdictional claims in published maps and institutional affiliations.

\section{Submit your manuscript to a SpringerOpen ${ }^{\circ}$ journal and benefit from:}

- Convenient online submission

Rigorous peer review

- Open access: articles freely available online

- High visibility within the field

- Retaining the copyright to your article

Submit your next manuscript at $\gg$ springeropen.com 